\title{
Diagnóstico endoscópico de estridor na infância*
}

\author{
Vítor EMANUEL CASSOL ${ }^{1}$
}

\begin{abstract}
Objetivo: Determinar os principais diagnósticos endoscópicos identificados em crianças que apresentam estridor, no Serviço de Pediatria do Hospital Universitário de Santa Maria. Métodos: Estudo descritivo, do tipo transversal, não controlado, com dados obtidos de protocolos preenchidos quando realizada a endoscopia respiratória, no período de março/93 a novembro/99. Resultados:

Foram realizadas 56 endoscopias respiratórias e identificadas 59 alterações compatíveis com o diagnóstico clínico de estridor, sendo $63 \%$ pacientes do sexo masculino. Lesões congênitas e adquiridas foram diagnosticadas em $45 \%$ e $50 \%$ dos casos, respectivamente, com $5 \%$ de endoscopias normais. Os diagnósticos mais comuns foram laringomalacia, estenose subglótica,

traqueobroncomalacia e edema de laringe. Conclusões: A investigação da via respiratória pela endoscopia demonstrou ser importante para o correto diagnóstico do estridor. Doenças congênitas ou adquiridas tiveram freqüência similar como causa de estridor. A investigação endoscópica revelou-se de grande valor, com elevado percentual de diagnóstico. (J Pneumol 2001;27(3):143-147)
\end{abstract}

\section{Endoscopic diagnosis of stridor in childhood}

Objective: To determine the main endoscopic diagnoses identified in children presenting stridor, at the Pediatric Unit of the General University Hospital of Santa Maria, Rio Grande do Sul, Southern Brazil. Methods: This is a cross sectional, uncontrolled, descriptive study. Data were obtained from the records of 56 consecutive patients submitted to bronchoscopy due to stridor; from March/93 to November/99. Results: Fifty-six bronchoscopies were performed and 59 lesions compatible with the diagnosis of stridor were observed. Patients were predominantly males (63\%). Congenital anomalies and acquired lesions were observed in $45 \%$ and $50 \%$ of the cases, respectively. The remaining $5 \%$ of the cases were normal. Laryngomalacia, subglottic stenosis, tracheobronchomalacia and laryngeal edema were the most common diagnoses.

Conclusions: The investigation of the respiratory tree by endoscopy is important for the correct diagnosis of children with stridor. Congenital or acquired diseases were similarly frequent as the cause of stridor. The endoscopic procedure was very valuable and yielded a high diagnostic rate.

Descritores - Toracoscopia. Diagnóstico. Sons respiratórios.

Key words - Thoracoscopy. Diagnosis. Respiratory sounds.

\section{INTRODUÇÃO}

O sinal clínico mais importante indicativo de obstrução das vias aéreas é o estridor e sua presença em uma criança requer avaliação completa da via respiratória(1). A pa-

* Trabalho realizado na Universidade Federal de Santa Maria, RS.

1. Especialista em Pneumologia Pediátrica pela SBP; Intensivista Pediátrico do HUSM-UFSM; Responsável pelo setor de Pneumologia Pediátrica do HUSM-UFSM.

Endereço para correspondência - Rua Pinheiro Machado, 2.350, Térreo, Edifício Central de Clínicas, Clínica de Crianças Miguel Meirelles 97050-600 - Santa Maria, RS. Tel. (55) 222-3033; fax (55) 2632208; E-mail: vitorcassol@conex.com.br

Recebido para publicação em 3/8/00. Reapresentado em 8/ 2/01. Aprovado, após revisão, em 20/2/01. lavra "estridor" é originária do termo latino stridulus, que quer dizer chiado, assobio, sibilo. O estridor é um sintoma audivel, produzido por um fluxo rápido e turbulento do ar, através de um segmento estreitado do trato respiratório, mais especificamente, as grandes vias aéreas. $\mathrm{O}$ tratamento adequado do estridor somente é possivel após correto diagnóstico ${ }^{(2)}$. O estridor é um sintoma e não um diagnóstico por si só, podendo ser causado por inúmeras condições e lesões ${ }^{(1-3)}$. Não é um achado comum, representando apenas $1 \%$ a $2 \%$ das internações em pediatria, mas sua gravidade como reflexo de uma doença possivelmente letal sobrepuja sua relativa raridade $e^{(3)}$. Para Wood ${ }^{(4)}$, uma das indicações mais comuns para exame endoscópico da via aérea em criança é o estridor, sendo a endoscopia (rígida e/ou flexível) na maioria das vezes um método rápido e conclusivo na avaliação das suas possiveis causas $^{(5)}$. Este estudo teve como objetivo avaliar, através de endoscopia respiratória realizada em um serviço de pe- 
diatria de um hospital geral universitário, as principais lesões que provocam estridor na criança.

\section{Casuística e método}

Foram analisados, prospectivamente, de março de 1993 a novembro de 1999 , protocolos de 56 pacientes pediátricos de ambos os sexos, submetidos a endoscopia respiratória rígida e/ou flexível, admitidos no serviço de pediatria do HUSM, que apresentaram sintomatologia de estridor ao ser admitidos no hospital ou durante seu período de internação.

O estudo foi do tipo transversal, não controlado, sendo os dados obtidos de protocolos preenchidos quando da realização da avaliação endoscópica respiratória com os seguintes dados: identificação, idade, sexo, condição clínica diagnosticada, diagnóstico endoscópico e complicações.

Os exames foram realizados no centro cirúrgico e unidades de terapia intensiva (neonatal ou pediátrica). Exames com broncoscópio rígido foram realizados sempre no centro cirúrgico sob anestesia geral inalatória, com xilocaína tópica a $1 \%$ e ventilação espontânea. Exames com broncoscópio flexível (em CC e/ou UTI) foram realizados com xilocaína tópica a $1 \%$ e sedação com morfina associada muitas vezes a diazepam ou midazolan.

Os dados foram tabulados e processados no programa Epi-Info versão 6.04.

\section{REsultados}

Foram realizadas 56 laringobroncoscopias em 56 crianças, sendo identificadas 59 lesões em via aérea causadoras de estridor. Em 15 pacientes (24\%) havia mais de uma lesão e, em seis $(9,7 \%)$, lesões em vias aéreas superiores $e$ inferiores.

A distribuição quanto a sexo e idade pode ser observado na Figura 1 e Tabela 1.

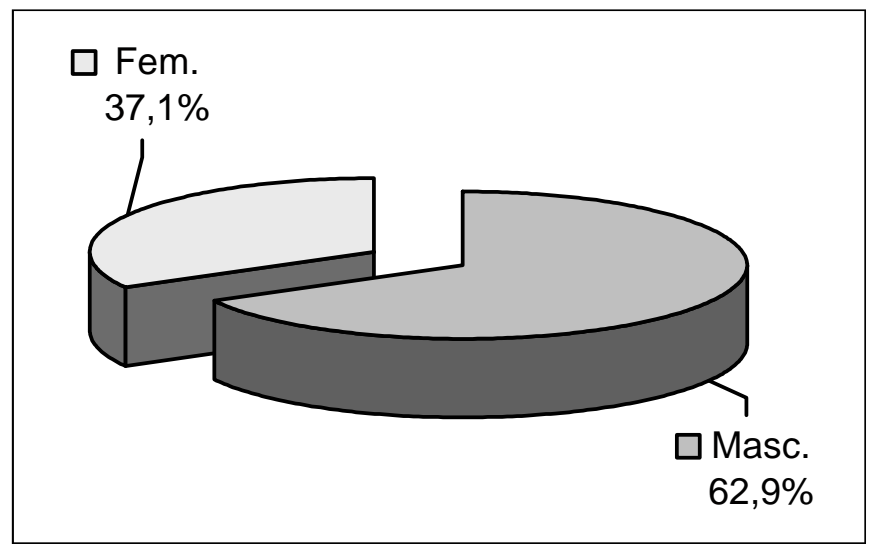

Figura 1 - Distribuição das crianças quanto ao sexo
Anomalias congênitas foram diagnosticadas como causa de estridor em 28 (45\%) dos pacientes. As lesões adquiridas (50\%) predominaram, tendo como condições determinantes intubação traqueal, prematuridade com necessidade de assistência ventilatória e desenvolvimento de displasia broncopulmonar, corpo estranho, lesão cáustica, refluxo gastroesofágico, pós-traqueostomia e causas infecciosas.

A relação dos achados endoscópicos está listada em ordem decrescente na Tabela 2. Como podemos observar, laringomalacia foi o diagnóstico mais freqüente, havendo percentual reduzido de achados normais.

\section{DISCUSSÃO}

A diversidade de alterações observadas endoscopicamente na via aérea superior neste estudo, principalmente em laringe e traquéia, demonstra a importância de realizar um correto diagnóstico do paciente com sintoma de estridor. A avaliação pela endoscopia respiratória deve ser realizada mesmo naquelas crianças com estridor inspiratório característico, não somente para confirmar o diagnóstico, mas principalmente para excluir outras causas $^{(6)}$.

TABELA 1

Distribuição das crianças quanto à idade

\begin{tabular}{crc}
\hline \multicolumn{1}{c}{ Idade } & $\mathbf{n}$ & $\boldsymbol{\%}$ \\
\hline$-\mathrm{I} \quad 1$ mês & 11 & 19,7 \\
$1-\mathrm{I} \quad 6$ meses & 23 & 41,0 \\
$6-\mathrm{I} 12$ meses & 4 & 7,1 \\
12 meses - & 18 & 32,1 \\
Total & 56 & 100 \\
\hline
\end{tabular}

TABELA 2

Distribuição das crianças quanto ao achado endoscópico

\begin{tabular}{lrr}
\hline \multicolumn{1}{c}{ Achado endoscópico } & $\mathbf{n}$ & \% \\
\hline Laringomalacia & 18 & 29 \\
Estenose subglótica & 8 & 12,9 \\
Traqueobroncomalacia & 8 & 12,9 \\
Edema de laringe & 5 & 8,1 \\
Paralisia de cordas vocais & 4 & 6,5 \\
Corpo estranho & 3 & 4,8 \\
Hemangioma subglótico & 2 & 3,2 \\
Membrana/banda em laringe & 2 & 3,2 \\
Laringotraqueobronquite & 2 & 3,2 \\
Outros* & 7 & 11,3 \\
Normal & 3 & 4,8 \\
Total & 62 & 100 \\
\hline * Estenose traqueal, membrana congênita laríngea, papilomatose, epiglotite, seqüela de le- \\
são cáustica, compressão da via aérea por linfangioma, tecido de granulação com flacidez \\
de aritenóide, mucopolissacaridose.
\end{tabular}


Em relação aos diagnósticos observados neste estudo, verifica-se um percentual elevado de lesões adquiridas como causa de estridor. Este achado pode ser explicado por duas razões: primeiro, faixa etária dos pacientes, com uma parcela significativa acima dos seis meses de idade, em que já predominam as lesões adquiridas como causa de estridor; segundo, muitas das crianças avaliadas eram pacientes das UTIs pediátrica e neonatal, geralmente avaliados após a intubação endotraqueal. Estudos mostram que grande parte de pacientes submetidos à intubação endotraqueal apresenta algum grau de lesão nas vias aéreas, quer a intubação tenha sido de curta ou longa duração(7). Fraga et al. ${ }^{(6)}$, no estudo das afecções envolvendo as regiões glótica e subglótica, demonstraram predominância de lesões congênitas, sendo a maioria dos pacientes na faixa dos primeiros meses de vida. Autores como Friedman et al. ${ }^{(8)}$ relataram predominância de lesões adquiridas como causa de estridor, provavelmente porque a idade de $76 \%$ dos seus pacientes avaliados era acima de um ano, em que a possibilidade de doenças congênitas é pequena.

Holinger(9) comenta em seu estudo que, se o estridor é de evolução gradual e presente desde os primeiros meses de vida, a etiologia geralmente é congênita. Nesse estudo, laringomalacia foi o diagnóstico mais freqüente. Demonstrou-se tal lesão em $30 \%$ dos casos investigados por estridor, constituindo $61 \%$ dos casos de lesões congênitas. Dickstein ${ }^{(10)}$, na avaliação endoscópica de 61 crianças com doença respiratória alta, diagnosticou laringomalacia em $26 \%$ dos casos, achado semelhante ao do presente estudo. Fraga et al. ${ }^{(6)}$, nas afecções envolvendo as regiões glótica e subglótica, identificaram laringomalacia em $60 \%$ dos casos. Estudos com grandes casuísticas, com alterações ao nivel de laringe, mostram, de modo semelhante, variação de 50 a $75 \%$ de laringomalacia( ${ }^{(6)}$. Autores como Maze e Bloch ${ }^{(11)}$ referem incidência de 68 a $75 \%$ de laringomalacia entre as doenças respiratória altas. Supomos que as diferenças em relação a maior ou menor incidência da doença, provavelmente, se deva às características peculiares de cada serviço.

Estenose subglótica foi a segunda causa de estridor mais freqüentemente diagnosticada. A diferenciação entre uma estenose subglótica congênita e adquirida, em geral, é difícil e com freqüência depende da existência de história pregressa de intubação endotraqueal ${ }^{(1)}$. A maioria dos casos de estenose subglótica foi considerada como adquirida pelo fato de os pacientes terem sido previamente intubados. Autores como Zalzal ${ }^{(12)}$ comentam que as últimas décadas assistiram a aumento da incidência de estenose subglótica em recém-nascidos prematuros, porque a traqueostomia foi substituída pela intubação para apoio das vias respiratórias; provavelmente, isso ocorra também na faixa etária dos primeiros anos de vida pela ne- cessidade de suporte ventilatório para muitas crianças internadas em unidades de terapia intensiva pediátrica.

Traqueomalacia ou traqueobroncomalacia foi outro achado freqüente na avaliação do estridor. Os que apresentaram traqueobrocomalacia eram, em sua maioria, pacientes com diagnóstico de atresia esofágica com fístula traqueoesofágica e prematuros submetidos a assistência ventilatória. Essa associação entre traqueomalacia e atresia de esôfago tem sido reconhecida por mais de duas décadas ${ }^{(13)}$, sendo que aproximadamente $30 \%$ das fístulas traqueoesofágicas estão associadas com traqueomalacia ${ }^{(14)}$. Piva et al. ${ }^{(14)}$ referem que a extensão do comprometimento na traqueomalacia é variável e freqüentemente está associada à fístula traqueoesofágica. Recémnascidos pré-termos submetidos à intubação prolongada $e$ ventilação mecânica podem também desenvolver traqueobroncomalacia ${ }^{(16)}$. Um dos pacientes avaliados em nosso estudo apresentava história de estridor com sibilos importantes, sendo diagnosticada traqueobroncomalacia primária com refluxo gastroesofágico. Esta criança vinha sendo tratada como asmática. Finder ${ }^{(16)}$, em revisão de broncomalacia primária em lactentes e crianças, relata que todos os 17 pacientes estudados haviam recebido inicialmente diagnóstico de hiperreatividade de via aérea. Traqueomalacia isolada pode não induzir sintomas obstrutivos importantes; todavia, muitas vezes apresenta-se associada a outras anomalias das vias aéreas, podendo complicar significativamente a dinâmica respiratória ${ }^{(1)}$.

Os pacientes que apresentaram estridor como manifestação de alteração (congênita ou adquirida) de corda vocal foram poucos $(6,5 \%)$, diferindo da literatura, que coloca paralisia de cordas vocais como segunda causa mais comum de estridor no neonato ${ }^{(17)}$. Dos quatro pacientes com lesão ao nível de corda vocal, dois casos foram de paralisia unilateral associada a trauma local (intubação), edema (refluxo gastroesofágico) e infecção, e um por laceração (com paresia) de corda vocal associada a estenose subglótica pós-intubação. Esses pacientes com paralisia unilateral apresentaram edema associado em região laríngea, o que explica a manifestação de estridor como sintoma importante, uma vez que paralisias unilaterais isoladas manifestam-se com sintomas respiratórios menos intensos ${ }^{(1)}$, usualmente produzindo choro fraco ${ }^{(17)}$. O paciente com paralisia bilateral de corda vocal necessitou de traqueostomia pela intensa dificuldade respiratória, não tendo sido encontrado o possivel fator causal para esta paralisia. Para Holinger et al. ${ }^{(18)}$, a maioria das paralisias de cordas vocais é idiopática e nenhuma outra anomalia pode ser detectada, ocorrendo freqüentemente resolução espontânea.

Edema de laringe como achado endoscópico foi responsável por $8 \%$ das lesões com manifestação clínica de estridor. Esses pacientes estiveram intubados para supor- 
te ventilatório e/ou apresentavam diagnóstico clínico de refluxo gastroesofágico. Fioretto et al. ${ }^{(19)}$, em estudo de complicações obstrutivas de vias aéreas pós-extubação, diagnosticaram edema de laringe pela laringoscopia em 9,5\% do total de 106 pacientes.

A relação do refluxo gastroesofágico com a doença laríngea foi primeiramente reconhecida por Cherry e Margulies no final da década de $60^{(20)}$. Esses pesquisadores reconheceram um padrão de dano laríngeo, com eritema e edema da aritenóide, mucosa pós-cricóide e cordas vocais posteriores ${ }^{(20)}$.

Corpos estranhos comprometendo a via aérea com manifestações clínicas de estridor foram responsáveis por $4,8 \%$ dos diagnósticos endoscópicos. Para Ryckman e Rodgers $^{(1)}$ a aspiração de corpo estranho é uma causa importante de estridor e sibilos no paciente pediátrico. Schidlow e Smith ${ }^{(21)}$ salientam que a localização de corpos estranhos na via aérea superior, particularmente na laringe, pode causar intenso estridor agudo e angústia respiratória em uma criança previamente saudável. Nesse estudo, dois corpos estranhos estavam localizados em terço superior de esôfago comprimindo a laringe e um em nível laringotraqueal, sendo estes corpos estranhos radiopacos em dois casos.

Uma série de outras lesões, tais como hemangioma subglótico, membrana ou banda ao nivel de laringe, processo inflamatório laringotraqueobrônquico, estenose traqueal, membrana congênita laríngea, papilomatose, epiglotite, seqüela de lesão cáustica em laringe, higroma cístico comprimindo laringe, tecido de granulação com flacidez de aritenóide e mucopolissacaridose foram diagnosticados como causa de estridor. Para Holinger e Brown" ${ }^{(22)}$, os hemangiomas subglóticos são lesões incomuns, respondendo por $1,5 \%$ das anomalias laríngeas congênitas.

\section{REFERÊNCIAS}

1. Ryckman F, Rodgers BM. Obstrução das vias aéreas em crianças. Simpósio sobre cirurgia pediátrica II. Clín Pediátr Am Norte 1989;36: 1739-1763.

2. Holinger LD. Evaluation of stridor and wheezing in pediatric laryngology and bronchoesophagology. In: Holinger LD, Lusk RP, Green CG, eds. Philadelphia: Lippincott-Raven Publishers, 1997;41-48.

3. Edwards DK. A criança com estridor. In: Hilton SVW, Edwards DK, eds. Radiologia pediátrica. $2^{a}$ ed. Rio de Janeiro: Guanabara Koogan, 1996;41-77.

4. Wood RE. Spelungin in the pediatric airway exploration with the flexible fiberoptic bronchoscope. Pediatr Clin North Am 1984;31:785-799.

5. Wood RE, Postma D. Endoscopy of the airway in infants and children. J Pediatr 1988;1-6.

6. Fraga JC, Nogueira A, Palombini B. Endoscopia respiratória rígida em criança. J Pediatr 1994;70:105-109.

7. Priebe HJ, Henke W, Whyte JH. Effects of traqueal intubation on laryngeal acoustic waveforms. Anesth Analg 1988;67:219-227.
Pacientes submetidos à intubação traqueal por períodos prolongados podem apresentar hiperemia, edema, exsudato e hemorragia de mucosa; ainda, formação de membrana/banda ao nivel de laringe e/ou estreitamento circunferencial(23), lesões diagnosticadas em alguns dos pacientes investigados por estridor. Para Stern ${ }^{(24)}$, membrana laríngea congênita é uma lesão incomum e pacientes com mucopolissacaridose podem apresentar obstrução da via aérea pelo acúmulo de mucopolissacarídeos ${ }^{(25)}$. A papilomatose em geral aparece na primeira ou segunda infância sob a forma de rouquidão e estridor ${ }^{(12)}$, sendo papilomas laríngeos múltiplos uma das causas menos freqüentes de obstrução laríngea(26).

Apenas 5\% dos pacientes investigados apresentaram exame endoscópico normal. Uma dessas crianças teve posteriormente o diagnóstico de doença de Gaucher, na qual espasmo laríngeo fazia parte do quadro clínico. Noutra paciente, com episódios recorrentes de estridor, foi diagnosticado estridor psicogênico com componente atópico. Em apenas um paciente, não foi encontrada uma causa que pudesse explicar o estridor. A positividade do exame endoscópico para investigação do estridor na criança foi de 95,2\% dos casos avaliados, valor semelhante aos $96,9 \%$ encontrados por Wood ${ }^{(4)}$ e um pouco superior aos $83 \%$ obtidos por Dickstein ${ }^{(10)}$ para doença respiratória alta. As lesões adquiridas foram freqüentes, provavelmente porque intubação endotraqueal e suporte ventilatório são procedimentos cada vez mais utilizados em centros de tratamento intensivos.

Em conclusão, a investigação endoscópica da via aérea para a criança com estridor apresenta elevado percentual de positividade, demonstrando ser de grande valor para um correto diagnóstico. Ainda, a assertiva "o estridor é um sintoma e não um diagnóstico por si só" parece ser verdadeira.
8. Friedman EM, Willians M, Healy GB, McGill TG. Pediatric endoscopy: a review of 616 cases. Ann Otol Rhinol Laringol 1984;93:517-519.

9. Holinger LD. Etiology of stridor. Ann Otol Rhinol Laryngol 1980;89: 397-400.

10. Dickstein PJ. Broncoscopia flexível de fibra-ótica em pediatria. J Pediatr 1992;68:6-12.

11. Maze A, Bloch E. Stridor in pediatric patient. Anesthesiology 1979; 50:132-145.

12. Zalzal GH. Estridor e comprometimento das vias respiratórias. Atualização em otorrinolaringologia pediátrica. Clínicas pediátricas da América do Norte. $1^{a}$ ed. Rio de Janeiro: Interlivros, 1989;1465-1479.

13. Filler RMG, Rossello PJ, Lebowitz RL. Life-threatening anoxic spells caused by tracheal compression after repair of esophageal atresia: correction by surgery. J Pediatr Surg 1976;11:739-748.

14. Piva JP, Gazal CHA, Muller H, Garcia PD. Obstrução das vias aéreas superiores. In: Piva JP, Carvalho P, Garcia PC, eds. Terapia intensiva em pediatria. $4^{\text {a }}$ ed. Rio de Janeiro: Medsi, 1997;133-152. 
15. Benjamin B. Tracheomalacia in infants and children. Ann Otol Rhinol Laryngol 1984;95:438.

16. Finder JD. Primary bronchomalacia in infants and children. J Pediatr 1997;130:59-66.

17. Mancuso RF. Stridor in neonates. Pediatr Clin North Am 1996;43: 1339-1355.

18. Holinger LD, Holinger PC, Holinger PH. Etiology of bilateral abductor vocal cord paralysis: a review of 389 cases. Ann Otol Rhinol Laryngol 1976;85:428-436.

19. Fioretto JF, Moreira FL, Bonatto RC, et al. Complicações obstrutivas de vias aéreas pós-extubação. Rev Paul Pediatr 1996;14:153-157.

20. Rudolph CD. Gastroesophageal reflux and airway disorders. The pediatric airway: a interdisciplinary approach. In: Myer CM, Cotton RT, Shott SR, eds. Philadelphia: J.B. Lippincott, 1995;327-357.

21. Schidlow DV, Smith DS. Estridor e obstrução das vias aéreas superiores. In: Schidlow DV, Smith DS, eds. Doenças respiratórias em pediatria. Rio de Janeiro: Revinter, 1999;31-37.
22. Holinger PH, Brown WT. Congenital webs, cysts, laryngoceles and other anomalies of the larynx. Ann Otol Rhinol Laryngol 1976;76: 744.

23. Zuckerberg AL, Nichols DG. Airway management in pediatric critical care. In: Rogers MC, Nichols DG, eds. Textbook of pediatric intensive care. 3rd ed. Baltimore: Associate editor, 1996;51-76.

24. Stern RC. Congenital anomalies. In: Behrman RE, Kliegman RM, Arvin AM, eds. Nelson textbook of pediatrics. 15th ed. Philadelphia: WB Saunders, 1996;1198.

25. Andrews TM. Airway obstruction in craniofacial anomalies: In: Myer CM, ed. The pediatric airway. 2nd ed. Philadelphia: J.B. Lippincott, 1995;247-260.

26. Phelan PD, Landau LI, Olinsky A. Respiratory noises: In: Phelan PD, Landau LI, Olinsky A, eds. Respiratory illness in children. 3rd ed. Carlton: Blackwell Scientific Publications, 1990;89-107. 